\title{
Gain properties of doped GaAs/AIGaAs multiple quantum well avalanche photodiode structures
}

\author{
H. M. Menkara, B. K. Wagner, and C. J. Summers \\ Quantum Microstructures Laboratory, Georgia Tech Research Institule, Atlanta, Georgia 30332-0800
}

(Received 12 September 1994; accepted for publication 3 February 1995)

\begin{abstract}
A comprehensive characterization has been made of the static and dynamical response of conventional and multiple quantum well (MQW) avalanche photodiodes (APDs). Comparison of the gain characteristics at low voltages between the MQW and conventional APDs show a direct experimental confirmation of a structure-induced carrier multiplication due to interband impact jonization. Similar studies of the bias dependence of the excess noise characteristics show that the low-voltage gain is primarily due to electron ionization in the MQW-APDs, and to both electron and hole ionization in the conventional APDs. For the doped MQW APDs, the average gain per stage was calculated by comparing gain data with carrier profile measurements, and was found to vary from 1.03 at low bias to 1.09 near avalanche breakdown. 1995 American Institute of Physics.
\end{abstract}

NASA-CR-201102

Extensive studies have been performed on multiple quantum well (MQW) structures because of their potential applications in avalanche photodiodes (APDs) and tunneling devices.' The MQW structure was first proposed by Chin et $a l^{2}$ and Capasso et al. ${ }^{3}$ as a method to enhance the electron-hole ionization ratio $(k=\alpha / \beta)$ beyond that in bulk materials. Later, Blauvelt ${ }^{4}$ proposed the doped MQW structure to further increase $k$. The desire for high performance optical detectors has resulted in several MQW APD structures using different material systems in an attempt to optimize their gain, noise, and bandwidth characteristics.

In this letter, we present direct experimental evidence of the gain enhancement at low voltages due to the built-in structure in a doped MQW APD. Our results are in good agreement with theoretical models developed by Brennans for similar derivatives of the doped MQW APD. The conventional APD structures investigated had a $2.5 \mu \mathrm{m}$ GaAs intrinsic region, and the $\mathrm{MQW}$ structures consisted of ten sets of altemating layers of $\mathrm{GaAs}(500 \AA)$ and $A l_{0.42} \mathrm{Ga}_{0.58} \mathrm{As}$ $(500 \AA)$ with $1000 \AA$ periods. All APDs were composed of a $1 \mu \mathrm{m}$ Be-doped $\left(3 \times 10^{18} \mathrm{~cm}^{-3}\right) p^{+}$top layer, and a 1.5 $\mu \mathrm{m}$ Si-doped $\left(3 \times 10^{18} \mathrm{~cm}^{-3}\right) n^{+}$back layer. In the MQW devices, high electric fields were achieved in the narrow band gap GaAs wells of the avalanche region through the introduction of thin (50 $\AA$ ) and highly doped (1.5 $\left.\times 10^{18} \mathrm{~cm}^{-3}\right) p^{+}$and $n^{+}$layers. Of the carriers that are photogenerated in the top $p^{+}$GaAs layer and diffuse toward the junction, only the electrons are injected into the highfield avalanche region. When a reverse bias is applied, the combined effect of the applied electric field, the built-in field. and the conduction band offset enhances the jonization process of electrons in the GaAs. The holes, on the other hand, are subjected to a smaller valence band discontinuity, and are "cooled" in the undoped AlGaAs regions.

All devices were grown by molecular beam epitaxy (MBE) in a Varian Gen II system and were fabricated using standard photolithographic techniques. Figure 1 shows a cross section of a MQW APD where contact rings were used to provide a uniform electric field across the entire device. The conventional APD design was similar, but with the MQW region replaced by a $2.5 \mu \mathrm{m}$ intrinsic GaAs layer.
The devices were characterized under both light and dark conditions using current-voltage $(I-V)$, capacitancevoltage $(C-V)$, and noise measurements. Gain curves were calculated from the reverse bias $I-V$ measurements performed as a function of photon flux. Carrier concentrations and depletion width profiles were determined from the $C-V$ data using a one-side junction approximation.

The $I-V$ measurements were taken in the dark and under HeNe laser illumination. In order to ensure pure electron injection, the laser beam was focused through a microscope objective at the center of the $75 \mu \mathrm{m}$ diameter opening in the upper $p$-contact ring. Breakdown voltages, $V_{B}$ were estimated to be about $27 \mathrm{~V}$ for the doped MQW APD and $63 \mathrm{~V}$ for the conventional APD with corresponding dark currents, measured at $20 \%$ of the breakdown voltages, of about 10 and $100 \mathrm{pA}$, respectively.

The $C-V$ measurements were performed at $1 \mathrm{MHz}$ using an HP4277A LCZ meter. The $C-V$ data were than analyzed to calculate the depletion widths and carrier profiles for the two structures. The net carrier concentration for the conventional APD is shown in Fig. 2 as a function of the calculated depletion width. As the reverse bias is increased, the capacitance decreases to $0.8 \mathrm{pf}$, while the depletion width increases to about $2.6 \mu \mathrm{m}$ prior to breakdown around $63 \mathrm{~V}$. Note that the carrier concentration increases shaply as the depletion edge is extended into the doped contact region. The inset of Fig. 2 shows the corresponding plot for the dopedwell MQW device which had a breakdown voltage, of about $27 \mathrm{~V}$. This plot clearly shows evidence of the depletion of all ten stages in the MQW APD. The presence of the peaks in the carrier profile data is due to the unequal $p$ and $n$ doping concentrations in the wells. Note that the peak positions do not agree with the 1000 A period in the MQW structure. This discrepancy is due to the fact that carrier concentration profiles were calculated assuming a one sided depletion. ${ }^{6}$ In addition, the spatial resolution of the $C-V$ measurements was limited by the Debye length given by $L_{D}$ $=\sqrt{\left(k T \varepsilon_{s} / q^{2} N\right)}$, which is about $40 \AA$ at room temperature for a doping level of $n=1.5 \times 10^{18} \mathrm{~cm}^{-3}$. Since the thickness of the doped layers in the wells was of the same 


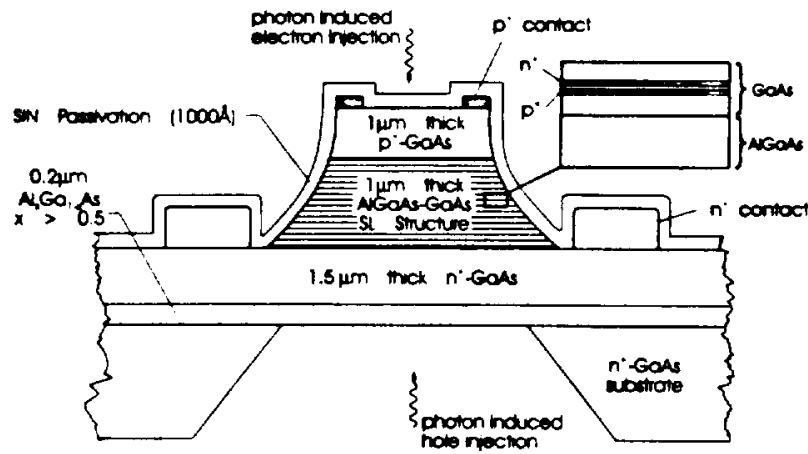

FIG. 1. Cross section of a MQW APD.

order of magnitude ( $50 \AA$ ), abrupt changes in the doping concentration could not be accurately measured.

The gain curves, calculated from the $I-V$ data, are shown in Fig. 3 where the bias values were normalized by the breakdown voltage of each device to enable comparison. Figure 3(a) clearly shows the presence of gain in the doped MQW device in the low voltage region while the conventional $p-i-n$ structure [Fig. 3(b)] does not show any gain in this regime. This is an indication of a structure-induced carrier multiplication resulting from the band discontinuity and the doping in the MQW APD.

In order to calculate the gain per period in the doped MQW, the carrier profile plot (inset of Fig. 2) was superimposed on the gain curve [Fig. 3(a)] and the gain was estimated at each consecutive concentration minimum. The corresponding gain values per stage were found to increase from 1.03 at low bias (one depleted stage), to about 1.09 near breakdown (ten depleted stages).

Multiplication noise measurements were also conducted on both APDs using an HP8568B spectrum analyzer set at a $200 \mathrm{kHz}$ center frequency with a $10 \mathrm{kHz}$ resolution bandwidths. The excess noise factor data are shown in Fig. 4 where the dashed lines represent McIntyre's calculated theoretical curves. McIntyre has shown ${ }^{7}$ that the statistical nature of the multiplication process adds an additional component to the noise which can be included with the shot noise of the APD as an excess noise factor. The excess noise factor in the case of pure electron injection is given by:

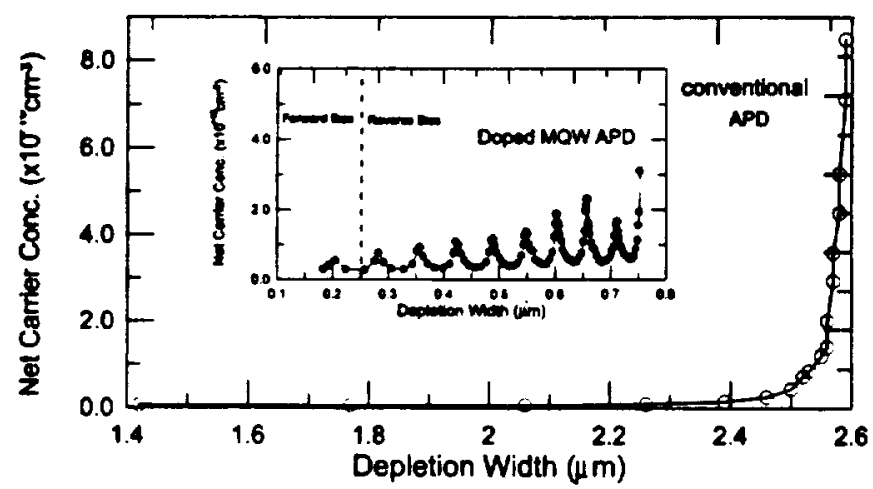

FIG. 2. Carrier concentration profile vs depletion width for the conventional APD and the doped MQW APD (inset).

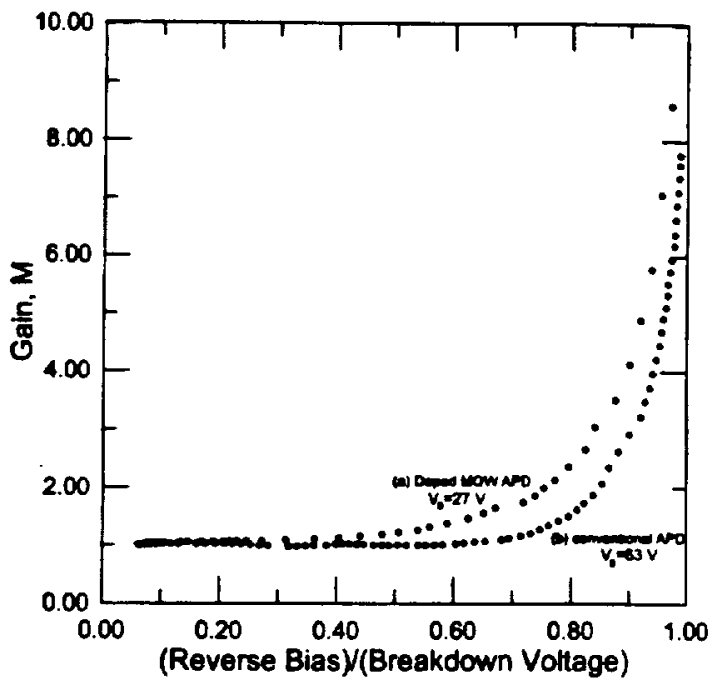

FIG. 3. Gain vs the ratio of the reverse bias to breakdown voluage for (a) the MQW APD and (b) the conventional APD.

$$
F_{e}=\frac{M}{k}+\left(1-\frac{1}{k}\right)\left(2-\frac{1}{M}\right),
$$

where $M$ is the multiplication factor, and $k$ is the effective electron to hole ionization ratio of the APD. It is important to note that the McIntyre model is not well suited for describing the noise characteristics of MQW devices, since it was intended mainly for conventional APDs. Better models had been developed and are described in the literature. ${ }^{8.9}$ However, McIntyre curves were used in our excess noise factor plot for comparison purposes in order to clearly illustrate the difference between the noise properties of both the conventional and the MQW APDs. Figure 4(a) for the doped MQW APD clearly shows that for low gains $(M<4)$, the ionization ratio is greatly enhanced $(k=10-50)$ as compared to that in bulk GaAs $(k=1.67)$. This fact is clear evidence of the validity of our previous results for the gain values per stage

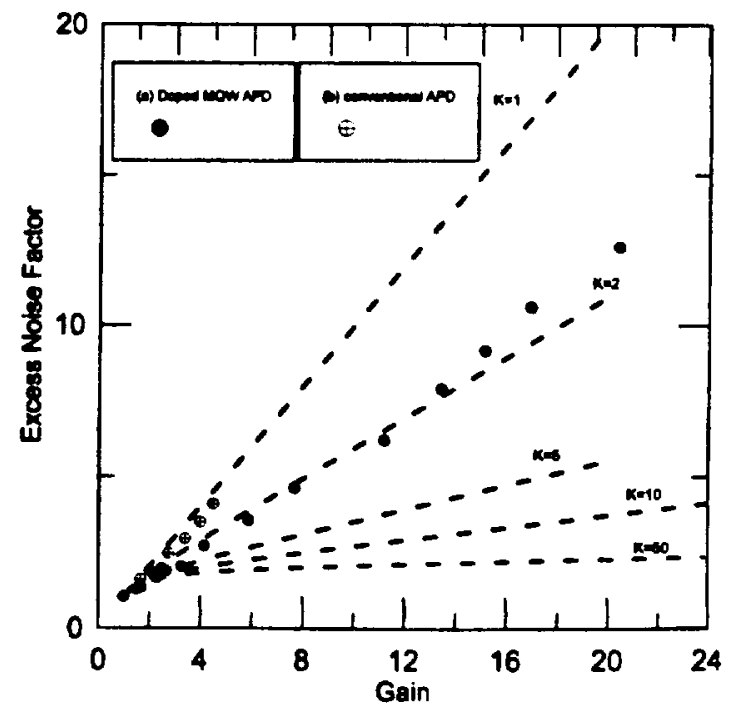

FIG. 4. Excess noise factor for both (a) the doped MQW APD and (b) the conventional APD. Dashed lines correspond to McIntyre's theoretical curves for $k=1,2,5,10$, and 50 . 
which assume single carrier multiplication at low voltages. At higher voltages, however, the value of $k$ is reduced since the holes gain more energy from the applied electric field and are more likely to impact ionize. ${ }^{10}$ The noise data for the conventional APD displayed in Fig. 4(b) show the high noise $(k \sim 1)$ characteristics of conventional APD even at low bias voltages. Excess noise factors at higher gain values were difficult to obtain since the dark current becomes large at high bias. Note that in Fig. 4(a), the point where the excess noise data break away from the high $k$ McIntyre curves corresponds to the breakdown voltage of the doped well APD. In addition, at high gains the $k$ ratio for the doped well APD approaches the bulk GaAs value of 1.67 as expected for high electric fields where the MQW structure is "washed out."

In summary, we have made a detailed comparison of the gain and noise characteristics of a conventional and a doped well MQW APD. The data obtained demonstrated a direct experimental evidence of structure induced preferential mul- tiplication of electrons over holes. As the bias was increased, the effect of the structure became less pronounced and the MQW device was reduced to a conventional structure.

'F. Capasso. Semiconductors and Semimetals, edited by R. K. Willardson and A. C. Beer (Academic, New York, 1985), Vol. 22, part D. p. 2.

${ }^{2}$ R. Chin, N. Holoniak, G. E. Stillman, J. Y. Tsang, and K. Hess, Appl. Phys. Lett. 16, 467 (1980).

${ }^{3}$ F. Capasso, W. T. Tsang, A. L. Hutchinson. and P. G. Williams, Appl. Phys. Lett. 40, 38 (1982).

${ }^{4} \mathrm{H}$. Blauvelh S. Margalit, and A. Yariv, Electron. Lett. 18, 375 (1982).

${ }^{5}$ K. Brennan, IEEE J. Quantum Electron. QE-22, 1999 (1986).

${ }^{6} \mathrm{~N}$. Yamamato, K. Yokoyama. and M. Yamamato, Appl. Phys. Letr. 62.252 (1993).

${ }^{7}$ R. J. Mclntyre, IEEE Trans. Electron Devices ED-13, 164 (1966).

${ }^{8} \mathrm{M}$. Teich. K. Matsuo, and B. Saleh. IEEE J. Quantum Electron. QE-22. 1184 (1986).

${ }^{9}$ N. Hakim. B. Saleh, and M. Teich, IEEE Trans. Electron Devices ED-37, $599(1990)$.

${ }^{10} \mathrm{P}$. Arisuin, A. Torabi, A. K. Garrison, H. M. Harris, and C. J. Summers. Appl. Phys. Lett. 60, 85 (1992). 